\title{
Stochastic Volatility Effects on Correlated Log-Normal Random Variables
}

\author{
Yong-Ki Ma \\ Department of Applied Mathematics, Kongju National University, Chungcheongnam-do 32588, Republic of Korea \\ Correspondence should be addressed to Yong-Ki Ma; ykma@kongju.ac.kr
}

Received 12 July 2017; Revised 25 September 2017; Accepted 9 December 2017; Published 28 December 2017

Academic Editor: Antonio Scarfone

Copyright (c) 2017 Yong-Ki Ma. This is an open access article distributed under the Creative Commons Attribution License, which permits unrestricted use, distribution, and reproduction in any medium, provided the original work is properly cited.

\begin{abstract}
The transition density function plays an important role in understanding and explaining the dynamics of the stochastic process. In this paper, we incorporate an ergodic process displaying fast moving fluctuation into constant volatility models to express volatility clustering over time. We obtain an analytic approximation of the transition density function under our stochastic process model. Using perturbation theory based on Lie-Trotter operator splitting method, we compute the leading-order term and the first-order correction term and then present the left and right skew scenarios through numerical study.
\end{abstract}

\section{Introduction}

The multivariate log-normal distribution is a widely used stochastic model in social sciences. What is the probability of the sum or difference of log-normal random variables? The solution to this question has wide applications in many fields such as finance $[1,2]$, actuarial science $[3,4]$, and physics [5]. Especially, in physics, examples include wave transmittance in random media, dissipation rate of turbulence energy, and temporal fluctuations of some nonlinear systems. However, to the best of the author of this paper's knowledge, almost nothing is known about the question yet. So, finding analytical approximations to it is as important as an alternative approach (see [6] for details). The main advantage of the analytical approximation approaches compared to other numerical methods is that in general the first ones are much faster and precise at least under certain model parameter regime. In addition, analytic approximation formulas retain qualitative model information and preserve an explicit dependence of the results on the underlying parameters. Many approximate methods can be categorized by their scope into three classes: generally correlated [7-10], independent [11-14], and independent and identically distributed [15, 16]. The probability density function of the correlated log-normal especially is carried out to propose various approximations of the sum distribution. These approximations can be divided into two categories. The first method is approximated by another log-normal random variable. For example, the lognormal parameters are obtained by moment matching [7], the mean and the variance are computed recursively [17], and the shifted log-normal distribution [18]. A second method of these methods is introduced to construct efficient numerical integrations techniques such as a steepest-descent integration [19] and a Smolyak's algorithm [20].

In option pricing theory, it is well-known that constant volatility for the stock price in the Black-Scholes model [21] can hardly capture the accumulated empirical evidence in financial markets since the parameters contained in the model actually change over time. A main drawback in the assumption of this model lies in the flat implied volatility surface, which is contradictory to empirical results that the implied volatilities of the equity options exhibit the smile or skew curve, in particular, from the 1987 crash onward. Among those several methods of overcoming the above drawback and relaxing the assumption of the Black-Scholes constant volatility model, a variety of stochastic volatility models have been suggested to incorporate the volatility skew. Fast mean reversion especially is one of the notable features of volatility. For example, Fouque et al. [22] estimated that its volatility reverts to the mean with characteristic time about 1.5 days in high-frequency S\&P 500 index data. Also, Engle [23] and Bollerslev [24] introduced the family of autoregressive 
conditional heteroskedasticity and generalized autoregressive conditional heteroskedasticity, respectively, to describe the evolution of the volatility of the stock price in discrete time setting and showed that econometric tests of these models reject the assumption of constant volatility and find evidence of volatility clustering over time.

Based on these observations together with the renowned contribution of stochastic volatility formula to option pricing, we propose the following fast mean-reverting (FMR) model in which asset prices are conditionally log-normal and the volatility process is a positive increasing function of a FMR process:

$$
\begin{aligned}
& d\left(X_{1} \pm X_{2}\right) \\
& =\left(f_{1}(Y) \pm f_{2}(Y)\right)\left(X_{1} \pm X_{2}\right) d\left(W_{1} \pm W_{2}\right), \\
& d Y=\alpha(m-Y) d t+\beta g(Y) d W_{Y},
\end{aligned}
$$

where $X_{1}$ and $X_{2}$ are log-normal random variables and $W_{1}$, $W_{2}$, and $W_{Y}$ are standard Brownian motions correlated as follows:

$$
\begin{aligned}
& d\left\langle W_{1}, W_{2}\right\rangle_{t}=\rho_{12} d t \\
& d\left\langle W_{1}, W_{Y}\right\rangle_{t}=\rho_{1 Y} d t \\
& d\left\langle W_{2}, W_{Y}\right\rangle_{t}=\rho_{2 Y} d t
\end{aligned}
$$

with $-1 \leq \rho_{12}, \rho_{1 Y}, \rho_{2 Y} \leq 1$. Here, the parameter $\alpha$ measures the rate at which the ergodic Markov process $Y$ reaches its long-term mean value $m, g$ is a function, $\beta$ is a constant, and the correlations $\rho_{1 Y}$ and $\rho_{2 Y}$ control the slope of the skew of each underlying. We assume that $\alpha$ is large so that volatility is sufficiently fast mean-reversion when looked over the time scale of options Fouque et al. [22]. We do not specify the concrete forms of $f_{1}, f_{2}$, and $g$ since they will not play an essential role in the perturbation theory performed in this paper but the functions must satisfy a sufficient growth condition to avoid some kind of bad cases such as nonexistence of the moments of $Y$. This generalization is a clear advantage of this model in that the FMR model encompasses the models which reflect stylized facts such as a feedback effect between volatility and volatility of volatility.

\section{Asymptotic Analysis}

2.1. Problem Formulation. Now we will denote $x_{1} \pm x_{2}$ and $x_{10} \pm x_{20}$ as $x^{ \pm}$and $x_{0}^{ \pm}$, respectively, for simplicity. The probability distribution of the sum or difference of the two correlated log-normal distributions can be obtained by calculating the integral

$$
\begin{aligned}
u_{ \pm}\left(t, x^{ \pm} ; t_{0}, x_{0}^{ \pm}\right)= & \int_{0}^{\infty} \int_{0}^{\infty} d x_{1} d x_{2} \\
& \cdot u\left(t, x_{1}, x_{2} ; t_{0}, x_{10}, x_{20}\right) \\
& \cdot \delta\left(x^{ \pm}-x_{0}^{ \pm}\right),
\end{aligned}
$$

where $u$ is the joint probability distribution of the two lognormal random variables and $\delta\left(x^{ \pm}-x_{0}^{ \pm}\right)$is the Dirac delta function. Unfortunately, a closed-form representation for this probability distribution still does not exist.

FMR volatility enables us to make good use of perturbation theory. To end this, we use the small positive parameter $\epsilon$, which denotes the inverse of the rate of mean-reversion $\alpha$ (which is assumed to be large). We suppose $\beta=\nu \sqrt{2} / \sqrt{\epsilon}$, where the variance $\nu^{2}$ of the invariant distribution of $Y$ is a constant with respect to $\epsilon$. If rewriting (2) in terms of $\epsilon$, we have

$$
d Y=\frac{1}{\epsilon}(m-Y) d t+\frac{\nu \sqrt{2}}{\sqrt{\epsilon}} g(Y) d W_{Y} .
$$

This gives a singular perturbation problem with respect to the small parameter $\epsilon$, which furnishes us with analytic tractability. We define the scaled FMR (SFMR) model as the FMR model used with an first-order asymptotic method in this paper.

If we compare the Heston model [25], one of representative stochastic volatility models, with the SFMR model, we can get some strengths. The Heston model has five parameters required to be estimated from market data while the SFMR model has two. In addition, the Heston model has certain restrictions on the volatility functions $f_{1}, f_{2}$, and $g$ whereas the SFMR model does not have any.

2.2. Perturbation Theory Based on Lie-Trotter Operator Splitting Method. Perturbation theory as developed by Fouque et al. [22] is a methodology utilized to find an approximated solution when the original problem is difficult to solve by separating it into more easily solvable, simple parts. If we apply the Feynman-Kac formula, we find that $u_{ \pm}^{\epsilon}$ satisfies the following Kolmogorov backward equation:

$$
\begin{aligned}
& \mathscr{L}^{\epsilon} u_{ \pm}^{\epsilon}\left(t, x_{1}, x_{2}, y_{1} ; t_{0}, x_{10}, x_{20}, y_{0}\right)=0, \quad t<T, \\
& \mathscr{L}^{\epsilon}:=\frac{1}{\epsilon} \mathscr{L}_{0}+\frac{1}{\sqrt{\epsilon}} \mathscr{L}_{1}+\mathscr{L}_{2}, \\
& u_{ \pm}^{\epsilon}\left(t, x_{1}, x_{2}, y_{1} ; t_{0} \longrightarrow t, x_{10}, x_{20}, y_{0}\right)=\delta\left(x^{ \pm}-x_{0}^{ \pm}\right),
\end{aligned}
$$

where

$$
\begin{aligned}
\mathscr{L}_{0}= & \left(m-y_{0}\right) \frac{\partial}{\partial y_{0}}+v^{2} g^{2}\left(y_{0}\right) \frac{\partial^{2}}{\partial y_{0}^{2}}, \\
\mathscr{L}_{1}= & \nu \sqrt{2} f_{1}\left(y_{0}\right) x_{10} g\left(y_{0}\right) \rho_{1 Y} \frac{\partial^{2}}{\partial x_{10} \partial y_{0}} \\
& +v \sqrt{2} f_{2}\left(y_{0}\right) x_{20} g\left(y_{0}\right) \rho_{2 Y} \frac{\partial^{2}}{\partial x_{20} \partial y_{0}}, \\
\mathscr{L}_{2}= & \frac{\partial}{\partial t_{0}}+\frac{1}{2} x_{10}^{2} f_{1}^{2}\left(y_{0}\right) \frac{\partial^{2}}{\partial x_{10}^{2}}+\frac{1}{2} x_{20}^{2} f_{2}^{2}\left(y_{0}\right) \frac{\partial^{2}}{\partial x_{20}^{2}} \\
& +x_{10} x_{20} \rho_{12} f_{1}\left(y_{0}\right) f_{2}\left(y_{0}\right) \frac{\partial^{2}}{\partial x_{10} \partial x_{20}} .
\end{aligned}
$$

Here, $\mathscr{L}_{0}$ is the infinitesimal generator of the SFMR process $Y . \mathscr{L}_{1}$ contains the mixed partial derivatives due to the 
correlations of the two Brownian motions $W_{1}$ and $W_{Y}$ and $W_{2}$ and $W_{Y}$, respectively. $\mathscr{L}_{2}$ is the operator of a generalized version of the two-dimensional standard Brownian motion at the volatility levels $f_{1}\left(y_{0}\right)$ and $f_{2}\left(y_{0}\right)$ instead of constant volatilities, respectively.

Before we solve problem (6), we write a useful lemma about the centering (or solvability) condition on the Poisson equation related to the operator $\mathscr{L}_{0}$ as follows.

Lemma 1. If $\psi(y) \in C^{2}(\mathbb{R})$ which is a solution to the Poisson equation

$$
\mathscr{L}_{0} \chi(y)+\psi(y)=0
$$

exists, then the centering condition $\langle\psi\rangle=\int \psi(y)(1 /$ $\left.\sqrt{2 \pi v^{2}}\right) \exp \left[-(y-m)^{2} / 2 v^{2}\right]=0$ must be satisfied, where the notation $\langle\cdot\rangle$ is the average (or expectation) with respect to the invariant distribution (namely, $\left.\mathcal{N}\left(m, v^{2}\right)\right)$ of $Y$. Then, solutions of (10) are given by the form

$$
\chi(y)=\int_{0}^{\infty} \mathbb{E}^{y}[\psi(Y) \mid Y=y] d t
$$

Proof. See Fouque et al. [22].

Using the resultant partial differential equation (PDE) (6) and expanding $u_{ \pm}^{\epsilon}$ in powers of $\sqrt{\epsilon}$, one can approximate $u_{ \pm}^{\epsilon}$ to the sum of the leading term $u_{ \pm}^{(0)}$ and the first correction term $\sqrt{\epsilon} u_{ \pm}^{(1)}$ as follows:

$$
u_{ \pm}^{\epsilon} \approx u_{ \pm}^{(0)}+\sqrt{\epsilon} u_{ \pm}^{(1)}
$$

Since we focus on the first-order correction term for $u_{ \pm}^{\epsilon}$, we reset (12) with respect to $\sqrt{\epsilon} u_{ \pm}^{(1)}$ and denote it by $\tilde{u}_{ \pm}^{(1)}$. Using perturbation theory based on Lie-Trotter operator splitting method, $u_{ \pm}^{(0)}$ and $\widetilde{u}_{ \pm}^{(1)}$ must satisfy the following PDEs with boundary conditions, respectively:

$$
\begin{aligned}
& \left\langle\mathscr{L}_{2}\right\rangle u_{ \pm}^{(0)}\left(t, x_{1}, x_{2} ; t_{0}, x_{10}, x_{20}\right)=0 \\
& \text { with }\left(t, x_{1}, x_{2} ; t_{0} \longrightarrow t, x_{10}, x_{20}\right)=\delta\left(x^{ \pm}-x_{0}^{ \pm}\right) \text {, } \\
& \mathscr{L}_{2} \widetilde{u}_{ \pm}^{(1)}\left(t, x_{1}, x_{2} ; t_{0}, x_{10}, x_{20}\right) \\
& \quad=\left\langle\mathscr{L}_{1} \mathscr{L}_{0}^{-1}\left(\mathscr{L}_{2}-\left\langle\mathscr{L}_{2}\right\rangle\right)\right\rangle u_{ \pm}^{(0)}
\end{aligned}
$$$$
\text { with } \tilde{u}_{ \pm}^{(1)}\left(t, x_{1}, x_{2} ; t_{0} \longrightarrow t, x_{10}, x_{20}\right)=0
$$

and then one obtains the solutions $u_{ \pm}^{(0)}$ and $\tilde{u}_{ \pm}^{(1)}$ of the PDEs, respectively,

$$
\begin{aligned}
& u_{ \pm}^{(0)}=\frac{1}{x^{ \pm} \sqrt{2 \widetilde{\sigma}_{ \pm}^{2} \pi\left(t-t_{0}\right)}} \\
& \cdot \exp \left[-\frac{\left\{\ln \left(x^{ \pm} / x_{0}^{ \pm}\right)+(1 / 2) \widetilde{\sigma}_{ \pm}^{2}\left(t-t_{0}\right)\right\}^{2}}{2 \widetilde{\sigma}_{ \pm}^{2}\left(t-t_{0}\right)}\right],
\end{aligned}
$$

$$
\begin{aligned}
& \tilde{u}_{ \pm}^{(1)}=-(T-t)\left[V_{1} \frac{\partial^{3}}{\partial x_{10}^{3}}+V_{2} \frac{\partial^{3}}{\partial x_{20}^{3}}+V_{12} \frac{\partial^{3}}{\partial x_{10} x_{20}^{2}}\right. \\
& \left.+V_{21} \frac{\partial^{3}}{\partial x_{10}^{2} x_{20}}\right] u_{ \pm}^{(0)},
\end{aligned}
$$

where

$$
\begin{aligned}
\left\langle\mathscr{L}_{2}\right\rangle= & \frac{\partial}{\partial t_{0}}+\frac{1}{2} x_{10}^{2} \bar{\sigma}_{1} \frac{\partial^{2}}{\partial x_{10}^{2}}+\frac{1}{2} x_{20}^{2} \bar{\sigma}_{2} \frac{\partial^{2}}{\partial x_{20}^{2}} \\
& +\bar{\sigma}_{1} \bar{\sigma}_{2} \bar{\rho} x_{10} x_{20} \frac{\partial^{2}}{\partial x_{10} \partial x_{20}}, \\
\bar{\sigma}_{1}:= & \sqrt{\left\langle f_{1}^{2}\right\rangle}, \\
\bar{\sigma}_{2}:= & \sqrt{\left\langle f_{2}^{2}\right\rangle}, \\
\bar{\rho}:= & \frac{\rho_{12}\left\langle f_{1} f_{2}\right\rangle}{\bar{\sigma}_{1} \bar{\sigma}_{2}}, \\
\tilde{\sigma}_{+}:= & \frac{\sqrt{\bar{\sigma}_{1}^{2}+\bar{\sigma}_{2}^{2}+2 \bar{\sigma}_{1} \bar{\sigma}_{2} \bar{\rho}}}{2} \\
\tilde{\sigma}_{-}:= & \frac{\bar{\sigma}_{1}^{2}-\bar{\sigma}_{2}^{2}}{2 \sqrt{\bar{\sigma}_{1}^{2}+\bar{\sigma}_{2}^{2}-2 \bar{\sigma}_{1} \bar{\sigma}_{2} \bar{\rho}}}
\end{aligned}
$$

and the constant parameters $V_{1}, V_{2}, V_{12}$, and $V_{21}$ are defined as follows:

$$
\begin{aligned}
V_{1}= & \frac{\nu \rho_{1 Y} \sqrt{\epsilon} x_{10}^{3}}{\sqrt{2}}\left\langle f_{1} g \theta_{1}^{\prime}\right\rangle, \\
V_{2}= & \frac{\nu \rho_{2 Y} \sqrt{\epsilon} x_{20}^{3}}{\sqrt{2}}\left\langle f_{2} g \theta_{2}^{\prime}\right\rangle, \\
V_{12}= & \frac{\nu \rho_{1 Y} \sqrt{\epsilon} x_{10} x_{20}^{2}}{\sqrt{2}}\left\langle f_{1} g \theta_{2}^{\prime}\right\rangle \\
& +\nu \sqrt{2 \epsilon} \rho_{12} \rho_{2 Y} x_{10} x_{20}^{2}\left\langle f_{2} g \theta_{12}^{\prime}\right\rangle, \\
V_{21}= & \frac{\nu \rho_{2 Y} \sqrt{\epsilon} x_{10}^{2} x_{20}}{\sqrt{2}}\left\langle f_{2} g \theta_{1}^{\prime}\right\rangle \\
& +\nu \sqrt{2 \epsilon} \rho_{12} \rho_{1 Y} x_{10}^{2} x_{20}\left\langle f_{1} g \theta_{12}^{\prime}\right\rangle .
\end{aligned}
$$

See the Appendix for the relevant technique.

As a result, the distributions of the sum and difference of correlated log-normal random variables under SFMR model are shown to follow combining a shifted log-normal distribution with mixed partial derivatives of it. Also, all the original parameters are absorbed in the group parameters $V_{1}$, $V_{2}, V_{12}$, and $V_{21}$ and the present level $y$ of the hidden process $Y$ driving the fast time-scale volatility needs not be specified in the present approximation. 


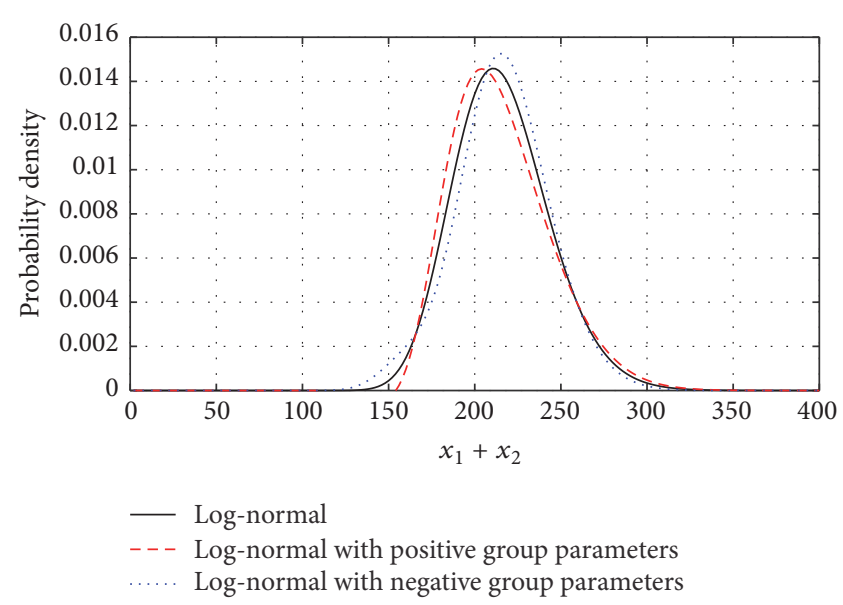

(a)

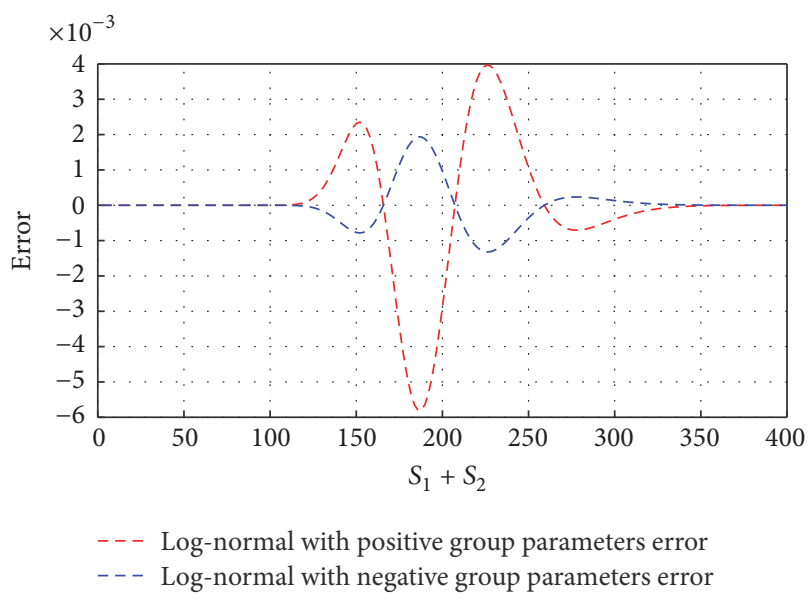

(b)

FIGURE 1: Sum of probability density function: $x_{10}=110, x_{20}=100, \bar{\sigma}_{1}=0.25, \bar{\sigma}_{2}=0.15, \bar{\rho}=-0.5$, and $t-t_{0}=1$.

2.3. Numerical Experiment. In this subsection, we illustrate the effectiveness of our result (12) by showing numerical results.

Several parameter sets are obtained from Lo except for group parameters. Figure 1(a) depicts log-normal distribution (leading-order term or Lo's result) and log-normal distributions under SFMR model with positive or negative group parameters (leading-order term plus first-order correction term), respectively. The solid line denotes the shifted lognormal distribution and the dash lines show combining the shifted log-normal distribution and the first correction term, respectively. Figure 1(b) depicts the errors calculated by subtracting the log-normal distributions with positive or negative group parameters from the log-normal distribution, respectively. We also apply the same result to the difference of the probability density function in Figure 2. Our numerical results show that the left and right skew scenarios are presented through first-order correction term and the major discrepancies appear around the peak of the probability density function. These pictures are sensitive to the choice of the involved parameters and give a lot of flexibility to the shape of the transition densities.

\section{Final Remarks}

Stochastic processes are popular in modeling various economics and financial variables. The transition density function especially plays a key role in the analysis of continuoustime diffusion models. In this paper, we obtained an analytic approximation of correlated log-normal random variables under SFMR model.

This paper offers various possible directions for further development. Our result can be applied to pricing and hedging spread options and is to incorporate a slowly varying volatility-driving process into the SFMR model. Also, this result can provide a very useful guide for credit risk management (see $[26,27])$. We leave these issues as future research topics.

\section{Appendix}

\section{Perturbative Analysis}

Now, we delineate the derivations of the PDEs (13) and (15).

We expand $u_{ \pm}^{\epsilon}$ in powers of $\sqrt{\epsilon}$ in order to apply the perturbation theory to the PDE problem (6):

$$
u_{ \pm}^{\epsilon}=u_{ \pm}^{(0)}+\sqrt{\epsilon} u_{ \pm}^{(1)}+\epsilon u_{ \pm}^{(2)}+\epsilon \sqrt{\epsilon} u_{ \pm}^{(3)}+\cdots
$$

Substituting (A.1) into (6), we reorganize the formula as follows:

$$
\begin{aligned}
& \frac{1}{\epsilon} \mathscr{L}_{0} u_{ \pm}^{(0)}+\frac{1}{\sqrt{\epsilon}}\left(\mathscr{L}_{0} u_{ \pm}^{(1)}+\mathscr{L}_{1} u_{ \pm}^{(0)}\right) \\
& \quad+\left(\mathscr{L}_{0} u_{ \pm}^{(2)}+\mathscr{L}_{1} u_{ \pm}^{(1)}+\mathscr{L}_{2} u_{ \pm}^{(0)}\right)+\cdots=0 .
\end{aligned}
$$

For (A.2) to hold for any $\epsilon>0$, each term of the equation must be zero. From the $1 / \epsilon$-order term, we obtain

$$
\mathscr{L}_{0} u_{ \pm}^{(0)}=0 .
$$

Since the operator $\mathscr{L}_{0}$ is the infinitesimal generator of the SFMR process $Y$, the solution $u_{ \pm}^{(0)}$ of the PDE must be a constant with respect to the variable $y ; u_{ \pm}^{(0)}=$ $u_{ \pm}^{(0)}\left(t, x_{1}, x_{2} ; t_{0}, x_{10}, x_{20}\right)$. From $1 / \sqrt{\epsilon}$-order term,

$$
\mathscr{L}_{0} u_{ \pm}^{(1)}+\mathscr{L}_{1} u_{ \pm}^{(0)}=0 .
$$

Since each term of the operator $\mathscr{L}_{1}$ contains $y$-derivative, the $y$-independence of $u_{ \pm}^{(0)}$ yields $\mathscr{L}_{1} u_{ \pm}^{(0)}=0$. The PDE (A.4) then reduces to $\mathscr{L}_{0} u_{ \pm}^{(1)}=0$, so that $u_{ \pm}^{(1)}$ is also independent of $y ; u_{ \pm}^{(1)}=u_{ \pm}^{(1)}\left(t, x_{1}, x_{2} ; t_{0}, x_{10}, x_{20}\right)$. Namely, the two terms $u_{ \pm}^{(0)}$ and $u_{ \pm}^{(1)}$ do not depend on the current level $y$ of the process $Y$ driving the fast scale volatility. One can continue to eliminate the terms of order $1, \sqrt{\epsilon}, \epsilon$, and so on. From the constant order terms, we have

$$
\mathscr{L}_{0} u_{ \pm}^{(2)}+\mathscr{L}_{1} u_{ \pm}^{(1)}+\mathscr{L}_{2} u_{ \pm}^{(0)}=0 .
$$



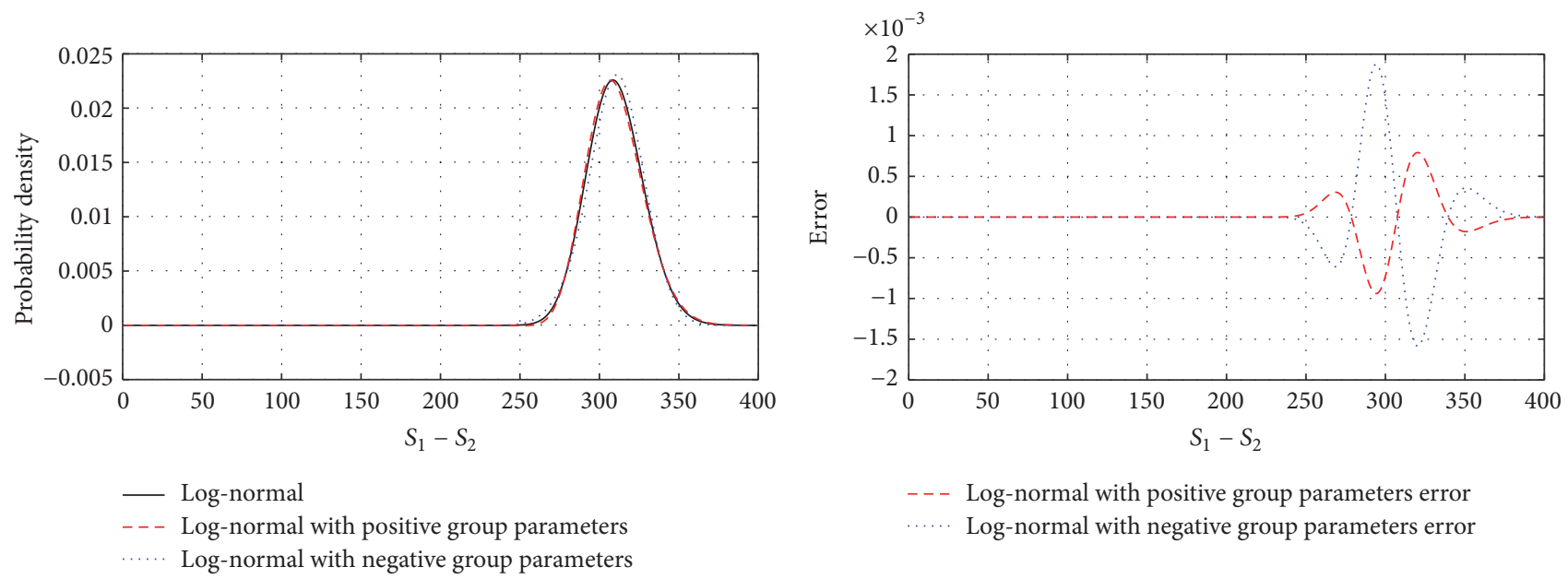

FIGURE 2: Difference of probability density function: $x_{10}=110, x_{20}=100, \bar{\sigma}_{1}=0.25, \bar{\sigma}_{2}=0.15, \bar{\rho}=-0.5$, and $t-t_{0}=1$.

This PDE becomes

$$
\mathscr{L}_{0} u_{ \pm}^{(2)}+\mathscr{L}_{2} u_{ \pm}^{(0)}=0
$$

due to the $y$-independence of $u_{ \pm}^{(1)}$ as seen above. The PDE (A.6) can be considered as a Poisson equation (see Lemma 1). From the centering condition with $\psi=\mathscr{L}_{2} u_{ \pm}^{(0)}$, the leadingorder $u_{ \pm}^{(0)}$ has to satisfy

$$
\left\langle\mathscr{L}_{2}\right\rangle u_{ \pm}^{(0)}=0
$$

where

$$
\begin{aligned}
\left\langle\mathscr{L}_{2}\right\rangle= & \frac{\partial}{\partial t_{0}}+\frac{1}{2} x_{10}^{2} \bar{\sigma}_{1} \frac{\partial^{2}}{\partial x_{10}^{2}}+\frac{1}{2} x_{20}^{2} \bar{\sigma}_{2} \frac{\partial^{2}}{\partial x_{20}^{2}} \\
& +\bar{\sigma}_{1} \bar{\sigma}_{2} \bar{\rho} x_{10} x_{20} \frac{\partial^{2}}{\partial x_{10} \partial x_{20}}, \\
\bar{\sigma}_{1}:= & \sqrt{\left\langle f_{1}^{2}\right\rangle}, \\
\bar{\sigma}_{2}:= & \sqrt{\left\langle f_{2}^{2}\right\rangle}, \\
\bar{\rho}:= & \frac{\rho_{12}\left\langle f_{1} f_{2}\right\rangle}{\bar{\sigma}_{1} \bar{\sigma}_{2}}, \\
\widetilde{\sigma}_{+}:= & \frac{\sqrt{\bar{\sigma}_{1}^{2}+\bar{\sigma}_{2}^{2}+2 \bar{\sigma}_{1} \bar{\sigma}_{2} \bar{\rho}}}{2}, \\
\widetilde{\sigma}_{-}:= & \frac{\bar{\sigma}_{1}^{2}-\bar{\sigma}_{2}^{2}}{2 \sqrt{\bar{\sigma}_{1}^{2}+\bar{\sigma}_{2}^{2}-2 \bar{\sigma}_{1} \bar{\sigma}_{2} \bar{\rho}}} .
\end{aligned}
$$

Then $u_{ \pm}^{(0)}$ solves the PDE (A.7).

Next, we derive the first correction term $u_{ \pm}^{(1)}$. From the $\sqrt{\epsilon}$-order term, we have

$$
\mathscr{L}_{0} u_{ \pm}^{(3)}+\mathscr{L}_{1} u_{ \pm}^{(2)}+\mathscr{L}_{2} u_{ \pm}^{(1)}=0
$$

Applying the centering condition with respect to $y$ to (A.9), we have

$$
\left\langle\mathscr{L}_{1} u_{ \pm}^{(2)}+\mathscr{L}_{2} u_{ \pm}^{(1)}\right\rangle=0 .
$$

From (9) and (A.7), we get

$$
\begin{aligned}
& \mathscr{L}_{2} u_{ \pm}^{(0)}=\mathscr{L}_{2} u_{ \pm}^{(0)}-\left\langle\mathscr{L}_{2} u_{ \pm}^{(0)}\right\rangle \\
& =\frac{1}{2} x_{10}^{2}\left(f_{1}^{2}-\left\langle f_{1}^{2}\right\rangle\right) \partial_{x_{10} x_{10}}^{2} u_{ \pm}^{(0)} \\
& \quad+\frac{1}{2} x_{20}^{2}\left(f_{2}^{2}-\left\langle f_{2}^{2}\right\rangle\right) \partial_{x_{20} x_{20}}^{2} u_{ \pm}^{(0)} \\
& \quad+x_{10} x_{20} \rho_{12}\left(f_{1}(y) f_{2}(y)-\left\langle f_{1} f_{2}\right\rangle\right) \partial_{x_{10} x_{20}}^{2} u_{ \pm}^{(0)}
\end{aligned}
$$

Substituting (A.12) into (A.6), we reorganize the form as follows:

$$
\begin{aligned}
u_{ \pm}^{(2)} & =-\mathscr{L}_{0}^{-1} \mathscr{L}_{2} u_{ \pm}^{(0)} \\
= & -\frac{1}{2} x_{10}^{2} \mathscr{L}_{0}^{-1}\left(f_{1}^{2}-\left\langle f_{1}^{2}\right\rangle\right) \partial_{x_{10} x_{10}}^{2} u_{ \pm}^{(0)}-\frac{1}{2} \\
& \cdot x_{20}^{2} \mathscr{L}_{0}^{-1}\left(f_{2}^{2}-\left\langle f_{2}^{2}\right\rangle\right) \partial_{x_{20} x_{20}}^{2} u_{ \pm}^{(0)} \\
& -x_{10} x_{20} \rho_{12} \mathscr{L}_{0}^{-1}\left(f_{1}(y) f_{2}(y)-\left\langle f_{1} f_{2}\right\rangle\right) \\
& \cdot \partial_{x_{10} x_{20}}^{2} u_{ \pm}^{(0)}=-\frac{1}{2} x_{10}^{2}\left(\theta_{1}(y)+a\left(t, x_{1}, x_{2}\right)\right) \\
& \cdot \partial_{x_{10} x_{10}}^{2} u_{ \pm}^{(0)}-\frac{1}{2} x_{20}^{2}\left(\theta_{2}(y)+b\left(t, x_{1}, x_{2}\right)\right) \\
& \cdot \partial_{x_{20} x_{20}}^{2} u_{ \pm}^{(0)}-x_{10} x_{20} \rho_{12}\left(\theta_{12}(y)+c\left(t, x_{1}, x_{2}\right)\right) \\
& \cdot \partial_{x_{10} x_{20}}^{2} u_{ \pm}^{(0)}
\end{aligned}
$$

for arbitrary finite-valued functions $a\left(t, x_{1}, x_{2}\right), b\left(t, x_{1}, x_{2}\right)$, and $c\left(t, x_{1}, x_{2}\right)$ where the functions $\theta_{1}: \mathbb{R} \rightarrow \mathbb{R}, \theta_{2}: \mathbb{R} \rightarrow \mathbb{R}$, 
and $\theta_{12}: \mathbb{R} \rightarrow \mathbb{R}$ are solutions of the Poisson equations, respectively:

$$
\begin{aligned}
& \mathscr{L}_{0} \theta_{1}=f_{1}^{2}-\left\langle f_{1}^{2}\right\rangle, \\
& \mathscr{L}_{0} \theta_{2}=f_{2}^{2}-\left\langle f_{2}^{2}\right\rangle, \\
& \mathscr{L}_{0} \theta_{12}=f_{1} f_{2}-\left\langle f_{1} f_{2}\right\rangle .
\end{aligned}
$$

From (A.11) and (A.13), we get

$$
u_{ \pm}^{(2)}=-\mathscr{L}_{0}^{-1}\left(\mathscr{L}_{2}-\left\langle\mathscr{L}_{2}\right\rangle\right) u_{ \pm}^{(0)} .
$$

Plugging (A.16) into (A.10), a PDE for $u_{ \pm}^{(1)}$ is given by

$$
\left\langle\mathscr{L}_{2}\right\rangle u_{ \pm}^{(1)}=\left\langle\mathscr{L}_{1} \mathscr{L}_{0}^{-1}\left(\mathscr{L}_{2}-\left\langle\mathscr{L}_{2}\right\rangle\right)\right\rangle u_{ \pm}^{(0)}
$$

Since we focus on the first-order correction term for $u_{+}^{\epsilon}$, we reset (A.17) with respect to $\sqrt{\epsilon} u_{ \pm}^{(1)}$ and denote it by $\widetilde{u}_{ \pm}^{(1)}$ so that the fast scale correction $\widetilde{u}_{ \pm}^{(1)}$ satisfies the following PDE:

$$
\begin{aligned}
\left\langle\mathscr{L}_{2}\right\rangle \widetilde{u}_{ \pm}^{(1)}=\mathscr{A} u_{ \pm}^{(0)} & \\
\mathscr{A} & :=\sqrt{\epsilon}\left\langle\mathscr{L}_{1} \mathscr{L}_{0}^{-1}\left(\mathscr{L}_{2}-\left\langle\mathscr{L}_{2}\right\rangle\right)\right\rangle .
\end{aligned}
$$

Here, the operator $\mathscr{A}$ is expressed as

$$
\begin{aligned}
\mathscr{A}= & V_{1} \partial_{x_{10} x_{10} x_{10}}^{3}+V_{2} \partial_{x_{20} x_{20} x_{20}}^{3}+V_{12} \partial_{x_{10} x_{20} x_{20}}^{3} \\
& +V_{21} \partial_{x_{10} x_{10} x_{20}}^{3},
\end{aligned}
$$

where the constant parameters $V_{1}, V_{2}, V_{12}$, and $V_{21}$ are defined as follows:

$$
\begin{aligned}
V_{1}= & \frac{\nu \rho_{1 Y} \sqrt{\epsilon} x_{10}^{3}}{\sqrt{2}}\left\langle f_{1} g \theta_{1}^{\prime}\right\rangle, \\
V_{2}= & \frac{\nu \rho_{2 Y} \sqrt{\epsilon} x_{20}^{3}}{\sqrt{2}}\left\langle f_{2} g \theta_{2}^{\prime}\right\rangle, \\
V_{12}= & \frac{\nu \rho_{1 Y} \sqrt{\epsilon} x_{10} x_{20}^{2}}{\sqrt{2}}\left\langle f_{1} g \theta_{2}^{\prime}\right\rangle \\
& +\nu \sqrt{2 \epsilon} \rho_{12} \rho_{2 Y} x_{10} x_{20}^{2}\left\langle f_{2} g \theta_{12}^{\prime}\right\rangle, \\
V_{21}= & \frac{\nu \rho_{2 Y} \sqrt{\epsilon} x_{10}^{2} x_{20}}{\sqrt{2}}\left\langle f_{2} g \theta_{1}^{\prime}\right\rangle \\
& +\nu \sqrt{2 \epsilon} \rho_{12} \rho_{1 Y} x_{10}^{2} x_{20}\left\langle f_{1} g \theta_{12}^{\prime}\right\rangle .
\end{aligned}
$$

It can be checked directly that $\tilde{u}_{ \pm}^{(1)}$ is given by

$$
\tilde{u}_{ \pm}^{(1)}=-(T-t) \mathscr{A} u_{ \pm}^{(0)} .
$$

\section{Conflicts of Interest}

The author declares that there are no conflicts of interest regarding the publication of this paper.

\section{Acknowledgments}

This work was supported by the National Research Foundation of Korea (NRF) grant funded by the Korean Government (MSIP) (no. 2015R1C1A1A01054663).

\section{References}

[1] M. A. Milevsky and S. E. Posner, "Asian options, the sum of lognormals, and the reciprocal gamma distribution," Journal of Financial and Quantitative Analysis, vol. 33, no. 3, pp. 409-422, 1998.

[2] R. Carmona and V. Durrleman, "Pricing and hedging spread options," SIAM Review, vol. 45, no. 4, pp. 627-685, 2003.

[3] J. Dhaene, M. Denuit, M. J. Goovaerts, R. Kaas, and D. Vyncke, "The concept of comonotonicity in actuarial science and finance: applications," Insurance: Mathematics and Economics, vol. 31, no. 2, pp. 133-161, 2002.

[4] S. Vanduffel, T. Hoedemakers, and J. Dhaene, "Comparing approximations for risk measures of sums of nonindependent lognormal random variables," North American Actuarial Journal, vol. 9, no. 4, pp. 71-82, 2005.

[5] M. Romeo, V. Da Costa, and F. Bardou, "Broad distribution effects in sums of lognormal random variables," The European Physical Journal B, vol. 32, no. 4, pp. 513-525, 2003.

[6] S. Nadarajah, "A review of results on sums of random variables," Acta Applicandae Mathematicae, vol. 103, no. 2, pp. 131-140, 2008.

[7] L. F. Fenton, "The sum of log-normal probability distributions in scatter transmission systems," IEEE Transactions on Communications Systems, vol. 8, no. 1, pp. 57-67, 1960.

[8] F. Berggren and S. B. Slimane, "A simple bound on the outage probability with lognormally distributed interferers," IEEE Communications Letters, vol. 8, no. 5, pp. 271-273, 2004.

[9] C. Lam and . Tho Le-Ngoc, "Outage probability with correlated lognormal interferers using log shifted gamma approximation," in Proceedings of the 2005 5th International Conference on Information Communications \& Signal Processing, pp. 618-622, Bangkok, Thailand.

[10] N. B. Mehta, A. F. Molisch, J. Wu, and J. Zhang, "Approximating the sum of correlated lognormal or lognormal-rice random variables," in Proceedings of the 2006 IEEE International Conference on Communications, ICC 2006, pp. 1605-1610, Turkey, July 2006.

[11] S. B. Slimane, "Bounds on the distribution of a sum of independent lognormal random variables," IEEE Transactions on Communications, vol. 49, no. 6, pp. 975-978, 2001.

[12] N. C. Beaulieu and Q. Xie, "An optimal lognormal approximation to lognormal sum distributions," IEEE Transactions on Vehicular Technology, vol. 53, no. 2, pp. 479-489, 2004.

[13] J. C. S. Santos Filho, M. D. Yacoub, and P. Cardieri, "Highly accurate range-adaptive lognormal approximation to lognormal sum distributions," IEEE Electronics Letters, vol. 42, no. 6, pp. 361-363, 2006.

[14] Z. Liu, J. Almhana, F. Wang, and R. McGorman, "Mixture lognormal approximations to lognormal sum distributions," IEEE Communications Letters, vol. 11, no. 9, pp. 711-713, 2007.

[15] N. C. Beaulieu and F. Rajwani, "Highly accurate simple closedform approximations to lognormal sum distributions and densities," IEEE Communications Letters, vol. 8, no. 12, pp. 709711, 2004. 
[16] L. Zhao and J. Ding, "A strict approach to approximating lognormal sum distributions," in Proceedings of the 2006 Canadian Conference on Electrical and Computer Engineering, pp. 916-919, Ottawa, ON, Canada, May 2006.

[17] S. C. Schwartz and Y. S. Yeh, "On the Distribution Function and Moments of Power Sums With Log-Normal Components," Bell System Technical Journal, vol. 61, no. 7, pp. 1441-1462, 1982.

[18] C. F. Lo, "The sum and difference of two lognormal random variables," Journal of Applied Mathematics, Art. ID 838397, 13 pages, 2012.

[19] D. Senaratne and C. Tellambura, "Numerical computation of the lognormal sum distribution," in Proceedings of the 2009 IEEE Global Telecommunications Conference, GLOBECOM 2009, December 2009.

[20] M. Di Renzo, L. Imbriglio, F. Graziosi, and F. Santucci, "Smolyak's algorithm: A simple and accurate framework for the analysis of correlated log-normal power-sums," IEEE Communications Letters, vol. 13, no. 9, pp. 673-675, 2009.

[21] F. Black and M. Scholes, "The pricing of options corporate liabilities," Journal of Political Economy, vol. 81, pp. 637-659, 1973.

[22] J.-P. Fouque, G. Papanicolaou, and K. Ronnie Sircar, Derivatives in Financial Markets with Stochastic Volatility, Cambridge University Press, 2000.

[23] R. F. Engle, "Autoregressive conditional heteroscedasticity with estimates of the variance of United Kingdom inflation," Econometrica, vol. 50, no. 4, pp. 987-1007, 1982.

[24] T. Bollerslev, "Generalized autoregressive conditional heteroskedasticity," Journal of Econometrics, vol. 31, no. 3, pp. 307327, 1986.

[25] S. L. Heston, "A closed-form solution for options with stochastic volatility with applications to bond and currency options," The Review of Financial Studies, vol. 6, no. 2, pp. 327-343, 1993.

[26] J.-H. Kim, Y.-K. Ma, and C. Y. Park, "Joint survival probability via truncated invariant copula," Chaos, Solitons and Fractals, vol. 85, pp. 68-76, 2016.

[27] Y.-K. Ma, "Modeling the dependency structure of integrated intensity processes," PLoS ONE, vol. 10, no. 8, 10 pages, 2015. 


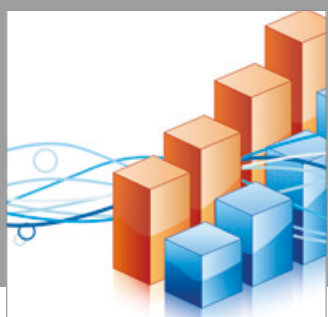

Advances in

Operations Research

vatersals

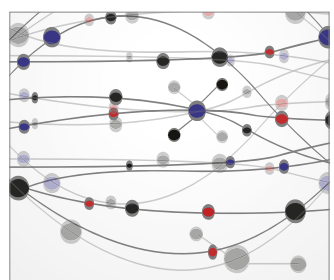

\section{The Scientific} World Journal
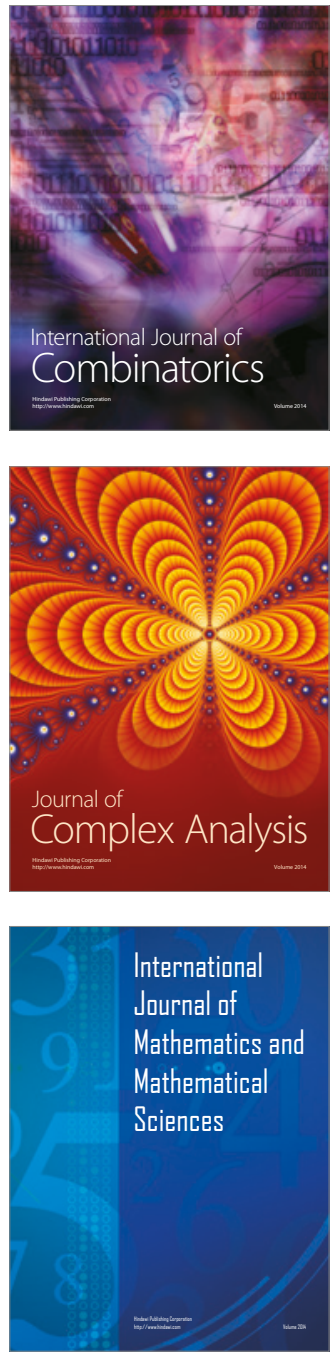
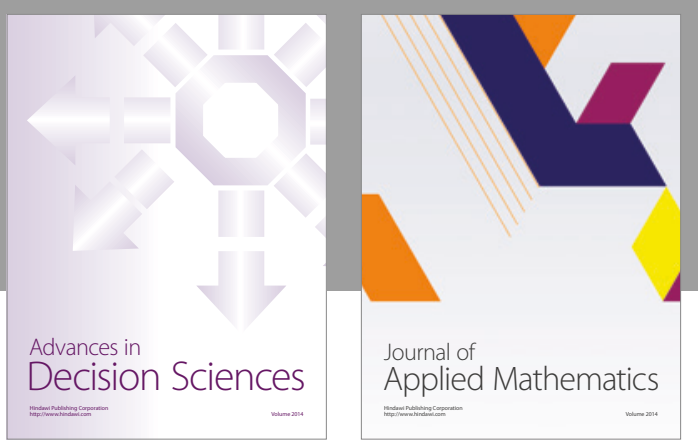

Algebra

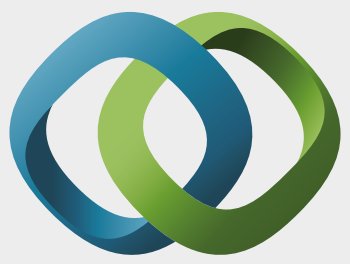

\section{Hindawi}

Submit your manuscripts at

https://www.hindawi.com
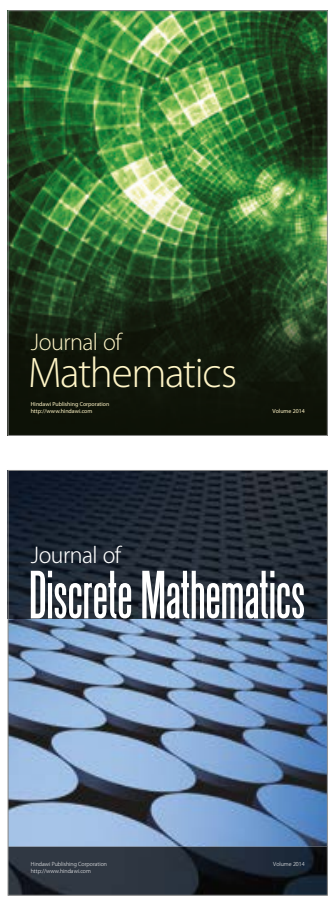

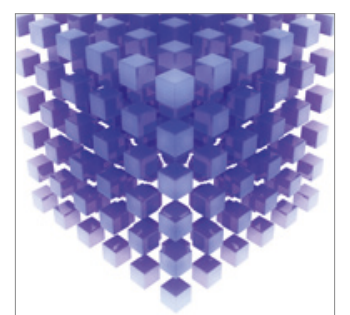

Mathematical Problems in Engineering
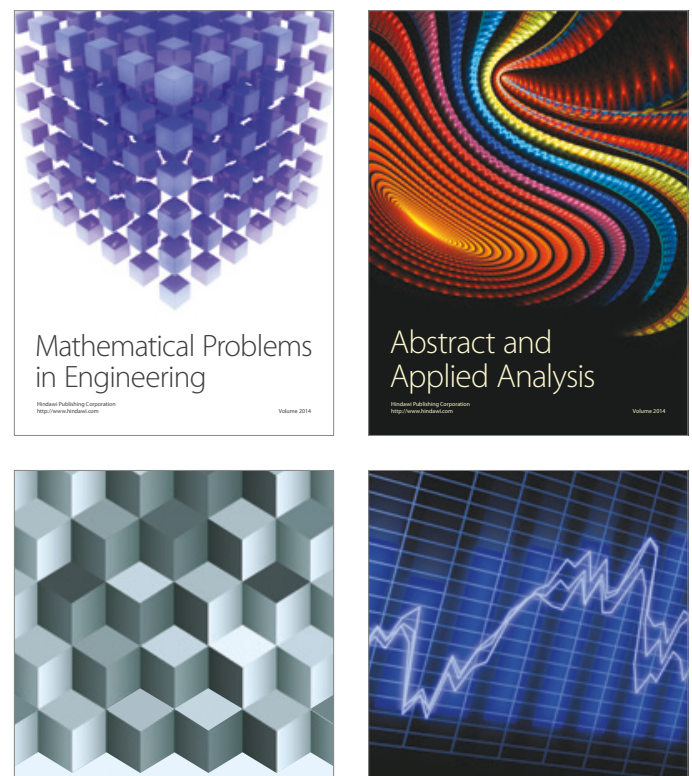

Journal of

Function Spaces

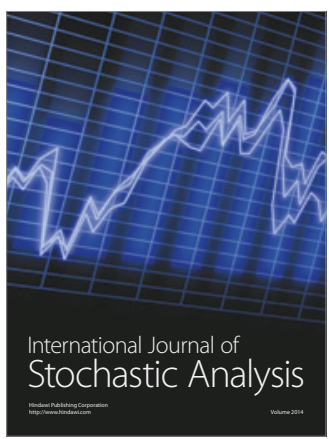

Probability and Statistics
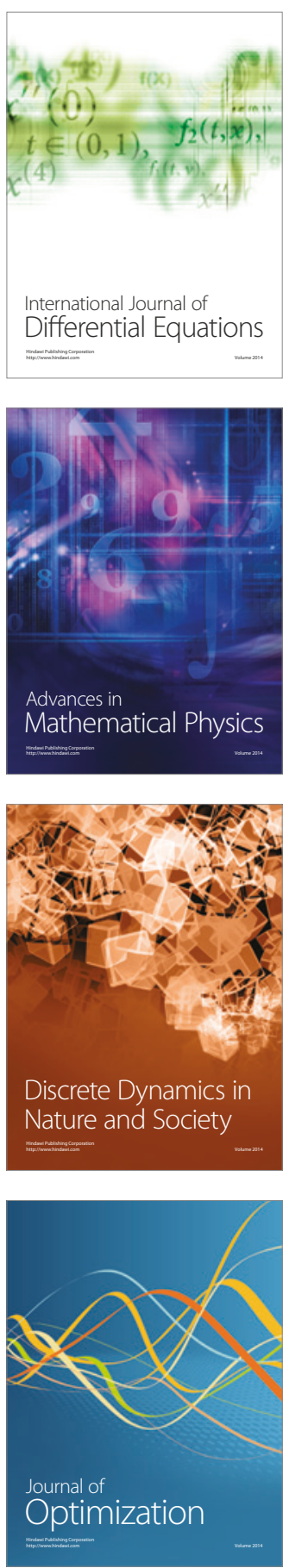